\title{
Ciclo errático de Dioctophyme renale: relato de dois casos
}

\author{
Cycle erratic Dioctophyme renale: report of two cases
}

Ciclo errático de Dioctophyme renale: relato de dos casos

\author{
Mayra A. Pereira- Figueiredo ${ }^{1}$, Daniela Franco-da Silva², Wilson Gómez- Manrique ${ }^{1}$, \\ Antônio A. Rodrigues- de Sousa ${ }^{3}$
}

1 Departamento de Patologia Veterinária, Faculdade de Ciências Agrárias e Veterinárias, Universidade Estadual Paulista (FCAV-Unesp), Via de Acesso Prof. Paulo Donato Castellane, s/n, Jaboticabal, SP 14884-900, Brasil.

2 Departamento de Reprodução Animal, Faculdade de Medicina Veterinária e Zootecnia, Universidade de São Paulo (FMVZ-USP), Av. Duque de Caxias Norte, Jardim Elite, Pirassununga, SP 13635 -900, Brasil.

3 Departamento de Clínica e Cirurgia, Curso de Medicina Veterinária, Universidade Estadual do Maranhão (UEMA), Cidade Universitária Paulo VI, Caixa Postal 09, Bairro Tirirical, São Luís, MA 65055-970, Brasil.

* Email: mayraaraguaia@hotmail.com

Recibido: febrero 15 de $2013 \quad$ Aceptado: mayo 14 de 2013

\section{Resumo}

O Dioctophyme renale é o maior nematelminto conhecido. A complexa cadeia epidemiológica deste se dá pelo ciclo evolutivo indireto que envolve diferentes espécies. Os ovos contendo larvas de primeiro estágio devem ser ingeridos por um anelídeo oligoqueta aquático, hospedeiro intermediário e essencial para a continuação do ciclo. Localiza-se no hospedeiro definitivo prioritariamente no rim direito, mas pode ser encontrado em localização ectópica, como a cavidade abdominal. Este trabalho relata a ocorrência de dois casos em São Luís do Maranhão, Brasil. No primeiro caso o animal tinha sido enviado ao Hospital Veterinário da Universidade Estadual do Maranhão para mastectomia e o segundo caso foi a uma consulta na clínica particular Veterinária Santo Antônio para verificação de um aumento de volume na região inguinal esquerda, achando nos dois casos, o nematóide $D$. renale. O presente relato contribui na importância se observar durante as cirurgias de cavidades a anatomia das vísceras e a presença de neoformações, no sentido do clínico-cirurgião se antecipar ao aparecimento de quaisquer tipo de sintomatologia clínica provocada por esse nematóide.

Palavras chave: ciclo ectópico, dioctofimose, nematelminto, parasita, tumor de mama

\begin{abstract}
Dioctophyme renale is the largest roundworm known. The complex epidemiological chain this is indirect life cycle that involves different species. The first-stage larvae-containing eggs must be ingested by an aquatic oligochaete annelid intermediate host, and essential for the continuation of the cycle. It is located in the definitive host priority in the right kidney, but can be found in ectopic location, such as the abdominal cavity. This paper reports the occurrence of two cases in São Luís do Maranhão, Brazil. In the first case the animal had been sent to the Veterinary Hospital of the State University of Maranhão for mastectomy and the second case was a consultation in private practice Veterinary Saint Anthony for verification of a volume increase in the inguinal region left, thinking in both cases, the nematode D. renale. The present report contributes to the importance of observing during cavity surgery the anatomy of the viscera
\end{abstract}


and the presence of in the direction of clinical surgeon to anticipate the emergence of any kind of clinical symptoms caused by this nematode.

key words: cycle ectopic, dioctophymosis, nematelminthe, parasite, breast tumor

\section{Introdução}

A infecção causada pelo Dioctophyme renale é conhecida como dioctofimose, apresenta-se comumente de forma assintomática, sendo feito o diagnóstico com base em achados acidentais em cirurgias e necropsias (Kommers et al., 1999). A maior frequência da doença é em cães não domiciliados e em canídeos silvestres, possivelmente por hábitos alimentares não seletivos (Mayrink et al., 2000).

Embora a descoberta do parasita seja bastante antiga, assim como sua descrição, a nomenclatura correta do parasita só foi padronizada em 1989, pela Comissão Internacional de Nomenclatura Zoológica, a qual finalizou os debates na escrita entre Dioctophyme ColletMeygret, 1982 ou, Dioctophyma Bosc, 1983, sendo favorável a primeira forma (Pedrassani, 2009; Verocai et al., 2009).

No Brasil a literatura descreve um maior número de casos em cães domésticos (Alencar Filho, 1966; Amato et al., 1976), sendo estes considerados hospedeiros definitivos anormais e terminais. Em animais silvestres há relatos em guaxinim (Procyon cancrivorus) (Kommers et al., 1999), preguiça (Choloepus didactylus) e furão (Galictis cuja) (Barros, 1990). Kommers et al., (1999) realizaram uma revisão sobre dioctiofimose em cães no Brasil onde citaram a ocorrência de $D$. renale em outros animais silvestres como o quati (Nasua nasua), a lontra (Lutra longicaudis) o lobo-guará (Chrysocyon brachiurus).

Esta doença é caracterizada como zoonose, o qual o homem é um dos hospedeiros definitivos, geralmente com localização na pele e nos rins, ocasionando cólicas renais e hematúria (Sujatha et al., 1983; Vibe, 1985). O homem, assim como os carnívoros, adquire esse parasito pela ingestão de carne crua de peixe e oligoquetas que albergam a forma larval infectante.

A complexa cadeia epidemiológica do $D$. renale se dá pelo ciclo evolutivo indireto que envolve animais aquáticos. Os ovos contendo larvas de primeiro estágio devem ser ingeridos por um anelídeo oligoqueta aquático (Lumbriculus variegatus), que é o único hospedeiro intermediário essencial para a continuação do ciclo (Barriga, 1982; Anderson, 1992). Após 100 dias de infecção do anelídeo, já pode ser encontrada a terceira forma larval (L3), infectante para os hospedei- ros definitivos e os paratênicos. Foi observado que nos hospedeiros paratênicos as larvas ficam encistadas, principalmente na parede do estômago, na musculatura abdominal e no mesentério, não evoluindo (Mace and Anderson, 1975). Os hospedeiros definitivos, geralmente, infectam-se ingerindo anelídeos oligoquetas ou hospedeiros paratênicos como crustáceos, peixes, sapos, rãs ou salamandras infectados (Osborne et al., 1969; Barriga, 1982; Anderson, 1992 Fortes, 1997, Ishizaki et al., 2010, Tokiwa et al., 2011). A rota migratória das larvas nos hospedeiros definitivos do estômago até o rim direito ainda é controversa. Sabe-se que no estômago a L3 muda para L4, daí pode penetrar no fígado ou ir para o duodeno, segue para a cavidade abdominal (onde ocorre a última muda) até chegar no rim direito. Os ovos depositados saem junto com a urina para o meio externo. Mas essa rota também é influenciada pela anatomia do hospedeiro, como foi observado em Mustela vison que possui uma proximidade entre o estômago, o lobo direito do fígado, a porção inicial do duodeno e o rim direito (Mace and Anderson, 1975). Nessa espécie ambos os sexos do parasita são encontrados no rim direito favorecendo a liberação de ovos férteis no ambiente, por isso, M. visoni é considerada como hospedeira definitiva verdadeira e reservatório do D. renal (Osborne et al., 1969). Nos cães é mais comum encontrar o parasita na cavidade abdominal, possivelmente porque os órgãos citados estão mais distantes (Hallberg, 1953).

O objetivo do presente trabalho é relatar dois casos de $D$. renale com ciclo errático, um encistado em tumor de mama de cadela e outro livre na região inguinal de um cão macho.

\section{Relato do primeiro caso}

Uma cadela sem raça definida (SRD), com oito anos de idade, não esterilizada e peso de $15 \mathrm{~kg}$, deu entrada no Hospital Veterinário da Universidade Estadual do Maranhão (UEMA) para realização de mastectomia na mama abdominal caudal esquerda. Realizou-se exame clínico e hemograma completo.

Foi realizado profilaxia antibiótica com enrofloxacina na dose de $5 \mathrm{mg} / \mathrm{kg}$ por via intramuscular (IM) e analgesia preemptiva com flunixin meglumine $1,1 \mathrm{mg} / \mathrm{kg} /$ IM. 
$\mathrm{Na}$ indução anestésica foi utilizada quetamina $5 \mathrm{mg} /$ $\mathrm{kg}$ associado ao diazepan 0,5 mg/kg por via intravenosa (IV). A anestesia foi mantida com halotano em circuito semi fechado. Foi administrada solução de ringer com lactato, $10 \mathrm{~mL} / \mathrm{kg} /$ hora durante todo o transoperatório.

Para a cirurgia de mastectomia foi realizada uma incisão na linha média na porção caudal. Após remoção do tumor encaminhou-se ao Laboratório de Anatomo-patologia/UEMA para tipificação do mesmo. Ao corte encontrou-se um helminto (Figura 1), e este foi enviado ao Laboratório de Parasitologia Veterinária/ UEMA, sendo identificado como um espécime macho de Dioctophyme renale de $19 \mathrm{~cm}$ de comprimento por $3 \mathrm{~cm}$ de largura.

\section{Relato do segundo caso}

Um cão SRD, com cinco meses de idade e peso de 8 $\mathrm{kg}$, oriundo do Bairro Ilhinha próximo à área de mangue, município de São Luís, foi encaminhado a Clínica Veterinária Santo Antônio para realização de uma consulta sob queixa principal de aumento de volume na região inguinal esquerda. Ao ser examinado optou-se por um procedimento cirúrgico exploratório. Administrando como profilaxia antibiótica, a base de penicilina benzatina $40 \mathrm{mg} / \mathrm{kg}$ IM e analgesia preemptiva o flunixin meglumine $1,1 \mathrm{mg} / \mathrm{kg} / \mathrm{IM}$.

$\mathrm{Na}$ anestesia foi utilizada quetamina $5 \mathrm{mg} / \mathrm{kg}$ associada à xilazina $1 \mathrm{mg} / \mathrm{kg} / \mathrm{IM}$ e manutenção anestésica com quetamina na metade da dose. Foi administrada solução de ringer com lactato $10 \mathrm{~mL} / \mathrm{kg}$ /hora durante todo o trans-operatório.

Feita a incisão no local onde apresentava o aumento de volume, encontrou-se um parasito identificado como Dioctophyme renale, fêmea de $33 \mathrm{~cm}$ de comprimento por $3 \mathrm{~cm}$ de largura (Figura 2).

\section{Discussão}

O ciclo do D. renale é indireto, tendo como hospedeiro definitivo os mustelídeos e canídeos; como hospedeiro intermediário, um anelídeo oligoqueta parasita de brânquias de peixe (Fortes, 1997).

Do primeiro relato o proprietário não afirmou que alimentou a cadela com peixe, no entanto, o cão do segundo relato tem livre acesso a rua e vive em um bairro cortado por mangues, com população de baixa renda e condições sanitárias precárias. Tendo esse animal oportunidades de se alimentar de sapos, rãs, peixes e crustáceos, hospedeiros paratênicos do $D$. renale. Esse fato corrobora com Pereira et al., (2006) que afirma que regiões com favorecimento hídrico aumenta a possibilidade de casos de dioctofimose, pelo fácil acesso dos animais aos hospedeiros paratênicos ou intermediários do nematódeo. Os peixes citados na literatura como hospedeiros paratênicos são os de água doce e diversas espécies já foram encontradas parasitadas (Pseudoscaphirynchus kaufmanni, Ictalurus nebulosus, Ictalurus melas, Esox lucius, Leuciscus idus, Aspius aspius, Rutilus rutilus, Chalcalburnus chalcoides, Pelecus cultratus, Barbus ranchicephalus, Gobio go-

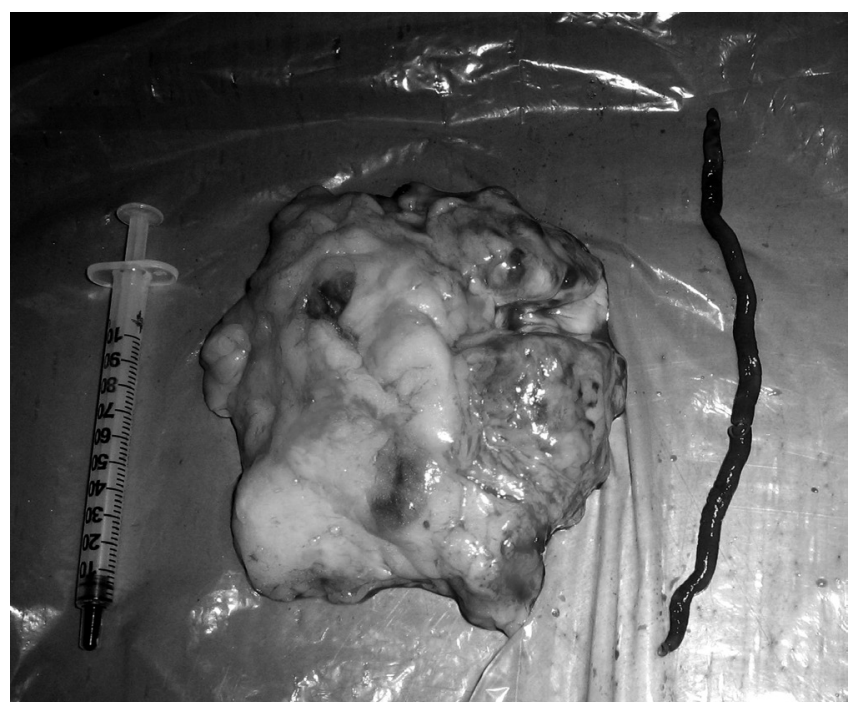

Figura 1. Dioctophyme renale, ao corte, retirado do tumor mamário abdominal caudal esquerdo de uma cadela. 


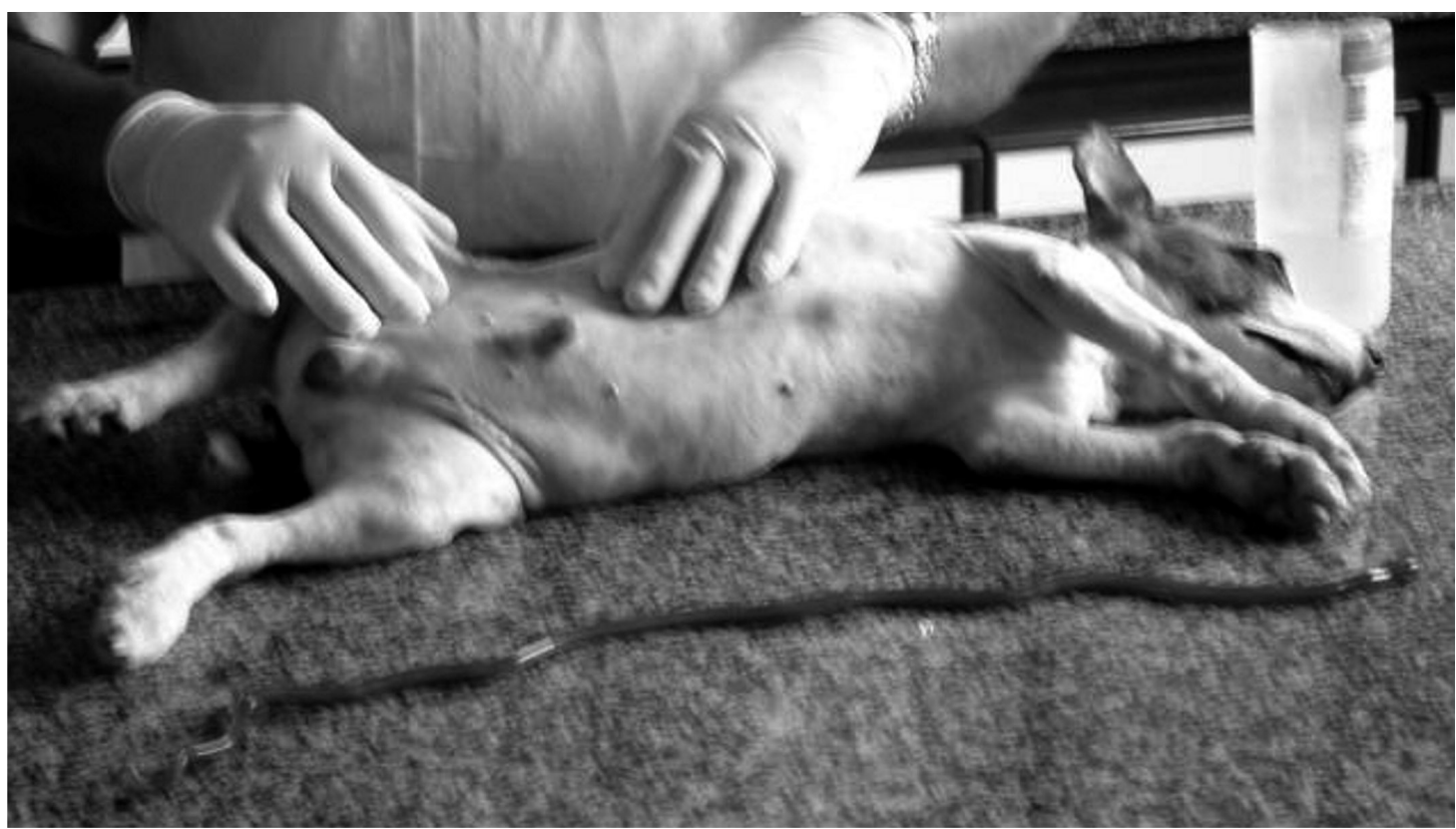

Figura 2. Dioctophyma renale, retirado do volume da região inguinal esquerda de um cão.

bio, Alburnoides taeniatus, Perca fluviatilis, Silurus glanis, Gambusia affinis, Lepomis gibbosus, Lepomis cyanellus e Micropterus salmoides) (Mace and Anderson, 1975; Measures and Anderson, 1985; Measures, 2001).

A dioctofimose ocorre mais frequentemente no rim direito dos hospedeiros definitivos, onde as fêmeas, quando fecundadas, produzem ovos que são eliminados pela urina (Amaral et al., 2008). A presença dos parasitos a nível ectópico não tem comprometimento macroscópico, diferente do que foi observado por Barros et al. (1990) e Pesenti et al., (2007) que relataram o envolvimento do rim direito de Galictis cuja chegando a produzir alterações microscópicas no rim parasitado (Leite et al., 2005). A presença do parasito no tecido mamário e subcutis da região inguinal não causaram alterações nem compressão de outros órgãos.

Conforme Eubanks e Pick (1963) e Mcleod (1967) os animais com dioctofimose são assintomáticos se somente um ou nenhum rim é parasitado. Nosso relato corrobora com os autores, pois os animais não apresentavam sintomatologia ao não possuir comprometimento dos rins. Porém, no cão do segundo relato iniciava-se um processo inflamatório caracterizado pelo aumento do volume na região inguinal. Os autores supracitados ainda afirmam que a cavidade abdominal pode se apresentar com bastante fibrina, característica de processo inflamatório causado pela presença do parasito, fato observado neste achado, ao redor do parasita localizado no subcutis.
Vários trabalhos relatam predomínio do parasita tanto no rim (lado direito) quanto na cavidade abdominal. Parasitas adultos têm sido encontrados principalmente nesses locais (Osborne et al., 1969; Amato et al., 1976; Hodesson et al., 1980; Barriga, 1982; Neves et al., 1983), entretanto, há divergência entre os autores quanto ao predomínio de um ou de outro local.

A região onde a larva infectante penetra no trato digestório do hospedeiro é determinante para a localização do parasito adulto, assim larvas infectantes que penetram na curvatura menor do estômago geralmente evoluem para a forma adulta entre os lobos hepáticos o que explica a forma livre do parasito nas cavidades corpóreas. Já aquelas que migram pela curvatura maior do estômago e duodeno, tendem a se alojar no rim esquerdo e direito respectivamente (Zabott et al., 2012). No presente relato, desconhecemos como foi a possível via de penetração para se localizarem na mama e na região inguinal.

Pereira et al., (2006) ao necropsiar um dos 67 cães do seu trabalho, encontrou uma massa no epíplon contendo $D$. renale, e em uma cadela encaminhada para cesariana um espécime na cavidade abdominal, indicando que este parasita pode ser encontrado em qualquer órgão ou cavidade, como foi observado nos presentes relatos. A localização ectópica dos parasitos adultos como observado neste relato, também foi descrita por Pesenti et al., (2007) e Zabott et al. (2012) em Galictis cuja e por Ishizaki et al., (2010) em Cebus 
apella. Apesar da fonte de infecção neste caso permanecer desconhecida, acreditamos que estes animais foram alimentados com peixes infectados.

Os achados clínicos associados à anamnese com relato da exposição do cão a um hospedeiro intermediário e, o encontro de ovos do D. renale são suficientes para se confirmar o diagnóstico, além da constatação e identificação de vermes por ocasião de necropsia (Fortes, 1997; Urquhart et al., 1998).

Os achados do exame físico podem permanecer normais ou pode-se encontrar um aumento de tamanho abdominal. Nas avaliações laboratoriais pelo hemograma pode-se observar eosinofilia, basofilia e hiperproteinemia; no bioquímico não ocorre azotemia a menos que ambos os rins sejam afetados ou um rim seja afetado e outro fique prejudicado devido a alguma outra causa. Na urinálise o diagnóstico deve ser confirmado por meio da observação de ovos característicos no sedimento urinário. Exige-se infestação renal para infecção patente. Podem ocorrer hematúria e proteinúria secundariamente a uma inflamação no trato urinário. Na análise de fluido abdominal, em casos de envolvimento peritoneal, os achados tornamse compatíveis com peritonite e os ovos do parasita podem ser encontrados no fluido abdominal (Birchard et al., 2003). Nos animais infectados com este parasita pode-se observar apatia, tristeza, emagrecimento, arqueamento do dorso, hematúria, aumento de volume palpável na região renal e às vezes, andar vacilante (Barriga, 1982). Além disso, pode-se observar peritonite e uremia devido à insuficiência renal (Fortes, 1997; Leite et al., 2005).

Em geral, para o diagnóstico da infecção causada por D. renale é constatado pela identificação de ovos em exame parasitológico de urina ou vermes por ocasião de necropsia. Às vezes vermes jovens podem ser eliminados pela urina (Fortes, 1997; Leite et al., 2005).

\section{Conclusão}

O presente trabalho relata o encontro de Dioctophyme renale em tumor mamário e a necessidade de durante as cirurgias de cavidades observar a anatomia das vísceras e a presença de neoformações, no sentido do clínico-cirurgião se antecipar ao aparecimento de qualquer sintomatologia clínica provocada por esse tipo de nematóide. Relata-se a ocorrência de parasitismo por Dioctophyme renale em cães domésticos no município de São Luís do Maranhão.

\section{Referências}

Alencar Filho RA. Dioctofimose renal em canino - Observação clínica. O Biológico. 1966; 33: 2-34.

Amaral LCD, Polizer KA, Sant'Ana TM. Neves MF. 2008. Dioctophyma renale. Revista Científica Eletrónica de Medicina Veterinária. 4(10). Publicação Científica da Faculdade de Medicina Veterinária e Zootecnia de Graça/FAMED. Disponível em <http://revista.inf.br/veterinaria08/revisao/13.pdf> Acessado em 13 janeiro. 2013.

Amato JFR, Grisi L, Miranda da Rosa VL. Reunião dos casos brasileiros de dioctofimose canina, com o registro do caso de mais alta intensidade de infecção por Dioctophyma renale (GOEZE, 1782). Rev Bras Biol, 1976; 36: 1-117.

Anderson RC. 1992. Nematode parasites of vertebrates - their development and transmission. Cambridge: CAB International, p. 533-539.

Barriga OO. 1982. Dioctophymosis. In: Schultz MG. CRC Handbook series in zoonoses. Florida: CRC. p. 83-92.

Barros DM, Lorini ML, Persson VG. Dioctophymosis in the little grison (Galictis cuja). J Wildl Dis, 1990; 26: 4-538

Birchard SJ, Sherding RG. Manual Saunders: Clínica de Pequenos Animais $2^{\circ}$ ed. São Paulo: Roca, 2003. p.207; 387.

Eubanks JW, Pick JR. Dioctophyma renale infection. J Am Vet Med Assoc, 1963; 143:164-169.

Fortes E. 1997. Parasitologia Veterinária. $3^{\underline{a}}$ ed. Editora Cone, São Paulo.

HALLBERG, C. W. Dioctophyma renale (Goeze, 1782) a study of the migration routes to the kidneys of mammals and resultant pathology. Trans Am Micros Soc, 1953; 72(4): 351-363.

Hodesson DL, Daniels RB, Richards B. Dioctophyma renale in a dog (a case report). Vet Med Small Anim Clin, 1980; 75(5): 825.

Ishizaki MN, Imbeloni AA, Muniz JAPC, Scalercio SRRA, Benigno RNM, Pereira WLA, Lacreta Junior ACC. Dioctophyma renale (Goeze, 1782) in the abdominal cavity of a capuchin monkey (Cebus apella), Brazil. Vet Parasitol, 2010; 173(3-4): 340-343.

Kommers GD, Ilha NRR, Barros CSL. Dioctofimose em cães: 16 casos. Ciência Rural, 1999; 29: 3-517.

Leite LC, Círio SM, Diniz JMF, Luz E, Navarro-Silva MA, Silva AWC, Leite SC, Zadorosnei AC, Musiat KC, Veronesi EM, Pereira CC. Lesões anatomopatológicas presentes na infecção por Dioctophyma renale (Goeze, 1782) em cães domésticos (Canis familiaris Linnaeus, 1758). Arch Vet Sci, 2005; 10(1): 95-101.

Mace TF, Anderson RC. Development of the giant kidney worm, Dioctophyma renale (Goeze, 1782) (Nematoda: Dioctophymatoidea). Departament of Zoology. Can J Zool, 1975; 53(11): 1552-1568.

Mayrink KC, Paes-de-Almeida EC, Thomé SMG. Dioctophyma renale (Goeze, 1782) em cães. Caderno Técnico Científico da Escola de Medicina Veterinária da Universidade do Grande Rio, Rio de Janeiro, 2000; 2: 20-40. 
Mcleod JA. 1967. Dioctophyme renale infections in Manitoba. Can J Zool, 45-505.

Measures LN. Dioctophymatosis. In: Samuel WM, Pybus MJ, Kocan AA. Parasitic Diseases of Wild Mammals. 2 ed. lowa State University Press: USA, 2001.

Measures LN, Anderson RC. Centrarchid fish as paratenic hosts of the giant kidney worm, Dioctophyma renale (GOEZE, 1782), in Ontario, Can J Wildl Dis, 1985; 21(1): 11-19.

Monteiro SG, Sallis ESV, Stainki DR. Natural infection by thirty four helminthes of the Dioctophyma renale (Goeze, 1782) em um cão. Revista da Faculdade de Zootecnia Veterinária e Agronomia, 2002; 9(1): 29-32.

Neves DS, Morais NA, Nogueira RHG, Chquiloff MAG. Ocorrência de Dioctophyme renale (Goeze, 1782) em cães da região de Lages, Estado de Santa Catarina. Arq Brás Méd Vet Zoot, 1983; 35(5): $665-673$.

Osborne CA, Stevenes JB, Hanlon GF, Rosin E, Bemrick WJ. Dioctophyma renale in a dog. J Am Vet Med Assoc, 1969; 155(4):605620 .

Pereira BJ, Girardelli GL, Trivilin LO, Lima VR, Nunes LC, Martins IVF. The occurrence of dioctophymosis in dogs from Municipality of Cachoeiro do Itapemirim in the State of Espírito Santo, Brazil, from May to December of 2004. Rev Bras Parasitol Vet, 2006; 15(3): 123-125.

Pesenti TC, Krüger C, Mascarenhas CS, Müller G. 2007. Ocorrência de Dioctophyma renale (Goeze, 1782) em Galictis cuja no Rio
Grande do Sul. XVI Congresso de Iniciação Científica UFPel, Pelotas.

Pedrassani D. Aspectos morfológicos, imunológicos e epidemiológicos do dioctophyme renale em cães o distrito de São Cristóvão, Três Barras, Santa Catarina. 2009. Tese de doutorado - Universidade Estadual Paulista, Faculdade de Ciências Agrárias e Veterinárias, 2009.

Sujatha SE, Fernando MBBS. The giant kidney worm (Dioctophyma renale) infection in man in Australia. Am J Surg Pathol, 1983 7(3): 281-284.

Tokiwa T, Harunari T, Tanikawa T, Akao N, Ohta N. Dioctophyme renale (Nematoda: Dioctophymatoidea) in the abdominal cavity of Rattus norvegicus in Japan. Parasitol Inter, 2011; 60(3): 324-326.

Urquhart GM, Armour J, Ducan JL, Dunn AM, Jennings FW. 1998 Parasitologia Veterinária. $2^{\circ}$ ed. Rio de Janeiro: Guanabara Koogan, p.86-87.

Verocai GG, Measures LN, Azevedo FD, Correia TR, Fernandes II, Scott, FB. Dioctophyme renale (Goeze, 1782) in the abdominal cavity of a domestic cat from Brazil. Vet Parasitol, 2009; 161: $342-344$.

Vibe PP. Dioctophyma infection in humans. Meditsinskaia Parazitoogiial. (Mosk), 1985; 1:83 - 84

Zabott MV, Pinto SB, Viott AM, Tostes RA, Bittencourt LHFB, Konell $\mathrm{AL}$, Gruchouskei L. Ocorrência de Dioctophyma renale em Galictis cuja. Pesq Vet Bras, 2012; 32(8): 786-788. 